\title{
What's New in Thyroid Oncology: Update from the European Society of Medical Oncology (ESMO) 2020 Annual Conference
}

\author{
Jochen H. Lorch
}

Department of Medical Oncology, Dana-Farber Cancer Institute, Harvard Medical School, Boston, Massachusetts, U.S.A.

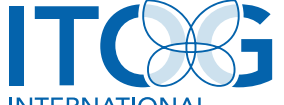

INTERNATIONAL THYROID ONCOLOGY GROUP
This review is part of an ongoing series marking the collaboration between the American Thyroid Association (ATA) and the International Thyroid Oncology Group (ITOG).
Two highlights mark the oncology calendar each year: the American Society of Clinical Oncology (ASCO) convention in May and its European equivalent, the European Society of Oncology (ESMO) meeting in September. While the ESMO meeting does not quite reach the level of the ASCO convention in size and scope, it is nonetheless an important conference at which new research data are presented. The other highlight with regard to the ESMO conventions are the locations, and this year was no exception (originally planned for Madrid); unfortunately, it became a victim of the COVID pandemic and was held as a virtual meeting. As at ASCO earlier this year, there was an unusual number of high-quality thyroid cancer presentations and interesting updates of studies that had been presented before.

One long-awaited study that was presented for the first time at ESMO 2020 was NRG-RTOG 0912, a randomized phase 2 study of radiation therapy and paclitaxel with pazopanib or placebo for anaplastic thyroid cancer by Eric Sherman and colleagues (1). Given the rarity and aggressiveness of anaplastic thyroid cancer, conducting and finishing a randomized study in this disease is a remarkable achievement. In this trial, a total of 89 patients were randomly assigned to receive radiation plus the radiation sensitizer paclitaxel with or without the multi-targeted tyrosine kinase inhibitor (TKI), pazopanib, based on in vitro data in this disease. The trial was designed to detect a $37.5 \%$ improvement in overall survival. Unfortunately, there was no significant difference between the two groups; although the 1-year survival in the experimental arm was an encouraging $37.1 \%$, as compared with $29 \%$ in the paclitaxel plus radiation control arm, the difference did not reach statistical significance $(P=0.283$ ). Nonetheless, the authors are to be congratulated for taking on this difficult-to-treat disease in a randomized study. In hindsight, the lack of convincing efficacy of another similar tyrosine kinase inhibitor, lenvatinib, could explain the lack of a more profound effect in this disease.

Or is it too early to dismiss the use of a multi-targeted TKI in anaplastic thyroid cancers? Dierks and colleagues studied 20 patients with poorly differentiated and anaplastic thyroid cancer treated with lenvatinib plus the PD-1 immune checkpoint inhibitor pembrolizumab prospectively and another 8 patients who were treated off protocol and evaluated retrospectively (2). Partial responses occurred in 6 of 16 patients (37.5\%) in the prospective data set, and a remarkable $66 \%$ complete response (CR) rate was observed in the retrospective analysis (4 of 6 patients). Given the obvious limitations of retrospective analysis and the small patient numbers particularly in the retrospective 
data review, it is difficult to draw meaningful conclusions from this experience, but these results seem encouraging. Could there be synergy between lenvatinib and PD1 inhibition after all?

To shed some light on this question, Haugen and colleagues examined whether the addition of pembrolizumab to lenvatinib (in cases in which lenvatinib therapy previously failed) could salvage the regimen and lead to a positive response (3). Expecting a definitive answer from this relatively small phase 2 study with 27 patients would be overly ambitious. The response rate for patients for whom lenvatinib had previously failed was $15 \%$-higher than the $9 \%$ response seen with pembrolizumab alone, which might have been due to chance alone. It was encouraging that the regimen did not pose any unexpected challenges in terms of toxicities.

Two Chinese studies examined the multi-targeted TKIs anlotinib and apatinib in radioactive iodine (RAI) refractory thyroid cancer (4,5). Chi and colleagues conducted a randomized, double-blind, multicenter phase 2 trial in 113 patients (76 in the anlotinib arm and 37 in the placebo arm). The study met its end point, with a median progression-free survival (PFS) of 40.54 months $(95 \% \mathrm{Cl}, 28.29$ to not evaluable [NE]) in the anlotinib arm and 8.38 months (95\% Cl, 5.59-13.80) in the placebo arm $(P<0.0001)$. These are certainly impressive efficacy data. However, it does not appear that disease progression within a specified time frame was required prior to enrollment, and the median PFS of 8.38 months suggests that many patients may not have had rapidly progressive disease. This makes comparisons between the phase 3 studies with lenvatinib and sorafenib difficult, if not impossible as both trials required disease progression within roughly a year prior to enrollment and therefore selected for a more aggressive thyroid cancer population.
In a similarly designed phase 3 study, Lin and colleagues reported the results from 92 patients who were randomly assigned to apatinib $(n=46)$ or placebo $(n=46)(5)$. The planned interim analysis showed a median PFS of 22.21 months $(95 \% \mathrm{Cl}$, 10.91 to not reached) in the apatinib group and 4.47 months $(95 \% \mathrm{Cl}, 1.94-9.17)$ in the placebo group. No disease progression at study entry was required, but the study population appears to have had more aggressive disease than in the anlotinib study, with an adequately short PFS in the placebo arm. Both studies add to the growing list of similar multi-targeted TKIs that have clear activity in RAI-refractory thyroid cancer and may be useful in parts of the world where lenvatinib and sorafenib are not available or as an additional line of treatment after failure of another TKI or other treatment regimen.

Similar to multiple meetings over the past 2 or more years, updates of the RET inhibitors selpercatinib (formerly known as LOXO 292) and pralsetinib (BLU667) (LIBRETTO and ARROW trials, respectively $(6,7)$ were again well represented. With longer followup, the impressive response rates of these drugs are maintained, adding to the notion that the treatment standard for RET-mutant medullary and RET fusionpositive differentiated thyroid cancer has changed to a more effective and more well-tolerated standard.

While the virtual format lacked the thrill and excitement of the in-person meetings of the past, it is good to see progress happening in thyroid oncology on so many fronts and around the world, despite the challenges during these trying times.

This article was prepared or accomplished by the listed author in his personal capacity. The opinions expressed in the article are the author's own and do not reflect the view of the International Thyroid Oncology Group. 


\section{References}

1. Sherman E et al. 2020 Randomized phase II study of radiation therapy and paclitaxel with pazopanib or placebo: NRG-RTOG 0912. Presented at the ESMO Annual Convention, September 19-21, 2020.

2. Diercks et al. 2020 The lenvatinib/pembrolizumab combination induces long lasting and complete responses in patients with metastatic anaplastic or poorly differentiated thyroid carcinoma: Results from a retrospective study and first results from the prospective phase II ATLEP trial. Presented at the ESMO Annual Convention, September 19-21, 2020.

3. Haugen B et al. 2020 Pembrolizumab salvage addon therapy in patients with radioiodine-refractory (RAIR), progressive differentiated thyroid cancer (DTC) progressing on lenvatinib: Results of a multicenter phase II International Thyroid Oncology Group trial. Presented at the ESMO Annual Convention, September 19-21, 2020.

4. Chi Y et al. 2020 Anlotinib in locally advanced or metastatic radioiodine-refractory differentiated thyroid carcinoma: A randomized, double-blind, multicenter phase II trial. Presented at the ESMO Annual Convention, September 19-21, 2020.
5. Lin Y et al. 2020 A randomized multicentered phase III study to evaluate apatinib in subjects with locally advanced or metastatic radioactive iodine-refractory differentiated thyroid cancer. Presented at the ESMO Annual Convention, September 19-21, 2020.

6. Wirth L et al. 2020 Exploratory patient-reported outcomes among patients with RET-mutant medullary thyroid cancer in LIBRETTO-001: A phase 1/2 trial of selpercatinib (LOXO-292). Presented at the ESMO Annual Convention, September 19-21, 2020.

7. Hu M et al. 2020 Results from the registrational phase 1/2 ARROW trial of pralsetinib (BLU-667) in patients (pts) with advanced RET mutation-positive medullary thyroid cancer (RET+ MTC). Presented at the ESMO Annual Convention, September 19-21, 2020.

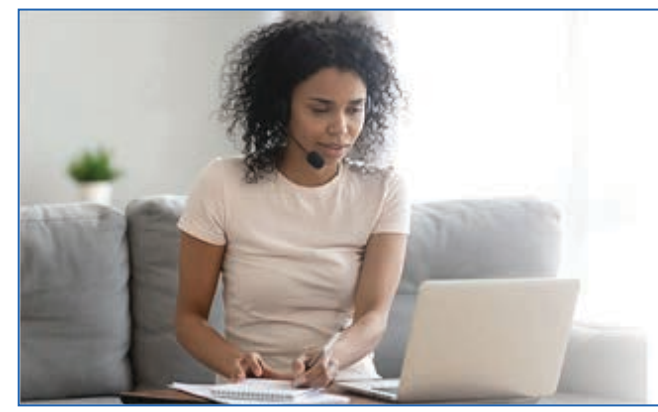

\section{American Thyroid Association ${ }^{\circ}$ 2020 Webinar Summer Series}

Join us for the ATA 10-webinar series from the convenience of your home or office. Some webinars include live Q\&A; All available on-demand.

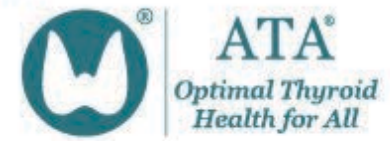

